\title{
Treatment of ventricular tachycardia-induced cardiomyopathy by transcatheter radiofrequency ablation
}

\author{
You-Ho Kim, Jeffrey Goldberger, Alan Kadish
}

\begin{abstract}
Catheter ablation of ventricular tachycardia was successfully performed in a patient with dilated cardiomyopathy (ejection fraction $38 \%$ ) and a long history of repetitive palpitations. Holter monitoring showed ventricular tachycardia that had a left bundle branch block QRS configuration with inferior axis deviation and was present for about one third of the daytime hours. At electrophysiological testing, ventricular tachycardia was reproduced by isoprenaline infusion. Radiofrequency energy delivered to the right ventricular outflow tract was successful at preventing the induction of ventricular tachycardia. Left ventricular ejection fraction had improved from $38 \%$ to $48 \%$ one month after ablation. During the follow up period of one year the patient remained free from arrhythmia on no medication. The ejection fraction was $61 \%$ one year after ablation. This report confirms that dilated cardiomyopathy can be induced by ventricular tachycardia and demonstrates that dilated cardiomyopathy can be reversed if the tachycardia is abolished by radiofrequency catheter ablation
\end{abstract}

(Heart 1996;76:550-552)

Keywords: ventricular tachycardia; radiofrequency; ablation; cardiomyopathy

Division of Cardiology, Northwestern

Memorial Hospital,

Northwestern

University Medical

School, Chicago,

Illinois, USA

Y-H Kim

J Goldberger

A Kadish

Correspondence to

Dr You-Ho Kim

Department of Medicine

Division of Cardiology, Asan

Medical Center and

University of Ulsan, 388-1

Poongnapdong, Songpagu,

Seoul 138-040, Korea.

Accepted for publication

25 June 1996

Tachycardia-induced cardiomyopathy associated with incessant supraventricular tachycardia has been well described. Curing the tachycardia can reverse left ventricular dysfunction. ${ }^{12}$ The mechanism of tachycardiainduced cardiomyopathy has not been completely defined, but animal studies suggested that changes in myocardial protein expression may be responsible for decreased left ventricular contractility. ${ }^{3}$ However, reports on the reversibility of tachycardia-induced cardiomyopathy are limited. ${ }^{45}$ Transcatheter radiofrequency (RF) ablation has been reported to be effective in abolishing ventricular tachycardia in patients with idiopathic ventricular tachycardia. ${ }^{6}$ We report the successful transcatheter ablation of ventricular tachycardia in a patient with dilated cardiomyopathy caused by repetitive monomorphic ventricular tachycardia in the right ventricular outflow tract. Ablation reversed left ventricular dysfunction.

\section{Case report}

A 42 year old man was referred for treatment of incessant ventricular tachycardia. The patient had an eight year history of intermittent palpitations predominantly associated with physical activity. In the months before admission, he developed exertional dyspnoea with ordinary activity. He had class II-III congestive heart failure. Holter monitoring showed almost incessant monomorphic ventricular tachycardia at rates of $210-240$ beats per minute which occurred only during the waking hours. Individual episodes of tachycardia lasted from 3 to 200 beats. The cycle length of the tachycardia was $300-320 \mathrm{~ms}$ and the QRS duration was $130 \mathrm{~ms}$. The 12 lead ECG showed ventricular tachycardia with a left bundle branch block configuration and an inferior axis (fig 1). Left ventriculography and coronary angiography showed a dilated left ventricle with diffuse, moderate dysfunction and normal coronary arteries. The ejection fraction calculated from a multiple gated (MUGA) blood pool scan was $38 \%$. Antiarrhythmic treatment with verapamil and atenolol reduced the frequency of ventricular tachycardia but did not completely suppress it.

\section{ABLATION PROCEDURE}

In the basal state there were frequent, isolated premature ventricular beats and frequent non-sustained ventricular tachycardia. Programmed ventricular stimulation with up to three extrastimuli at both the right ventricular apex and outflow tract failed to induce sustained ventricular tachycardia. When isoprenaline was given intravenously $(2 \mu \mathrm{g} / \mathrm{min})$ it markedly increased the frequency and duration of ventricular tachycardia. During ventricular tachycardia the earliest site of activation was localised at the right ventricular outflow tract along the posterior interventricular septum. A total of four radiofrequency energy $(25-30 \mathrm{~W}$ for $10-60 \mathrm{~s}$ ) lesions were delivered without 
Figure 1 Twelve lead electrocardiogram showing spontaneous ventricular tachycardia with a left bundle inferior axis configuration. There is some beat to beat variability in $Q R S$ morphology, which may in part be the result of fusion beats. The large time lines indicate one second intervals.
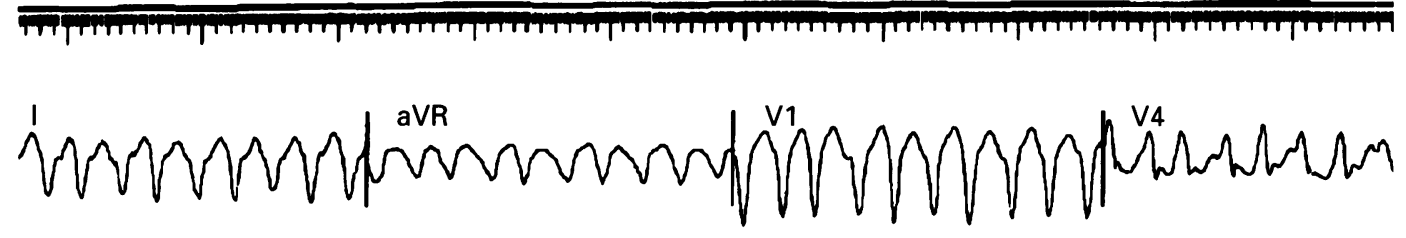

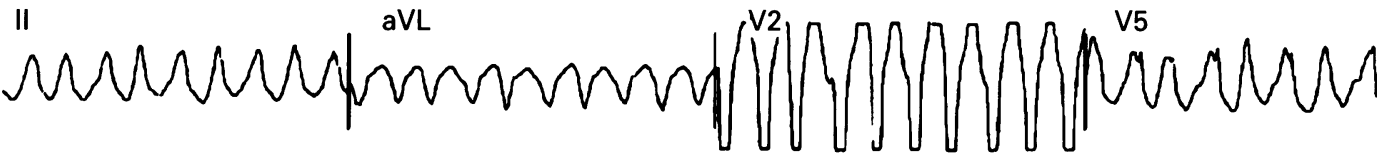

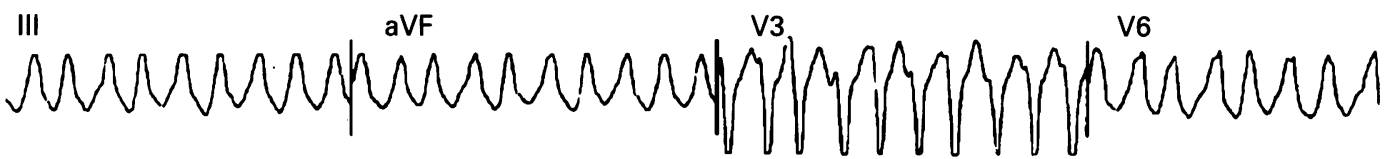

success to the posterior interventricular septum in the right ventricular outflow tract. The catheter was then advanced slightly and more superiorly and medially to just below the pulmonary valve. At this site, the catheter was advanced with firm pressure against the interventricular septum to maintain a stable position. As the catheter was advanced there was a sudden decrease in the frequency and duration of non-sustained ventricular tachycardia that resulted in periodic, isolated premature ventricular beats every five normal QRS complexes. Radiofrequency energy (30 W for $70 \mathrm{~s}$ ) was delivered to this region. After two seconds of radiofrequency energy delivery, premature

ventricular beats were abolished and did not recur. Thirty minutes after the last lesion was delivered, isoprenaline infusion (up to $3 \mu \mathrm{g} / \mathrm{min}$ ) and programmed cardiac stimulation did not induce premature ventricular beats or ventricular tachycardia.

A repeat blood pool scan four weeks after the procedure revealed a left ventricular ejection fraction of $48 \%$ and normal chamber sizes with nearly normalised wall motion of the left ventricle; the patient became asymptomatic. A Holter monitor did not show any premature ventricular beats over a 24 hour period. During one year of follow up, the patient remained free from recurrent palpitations on no antiarrhyth-

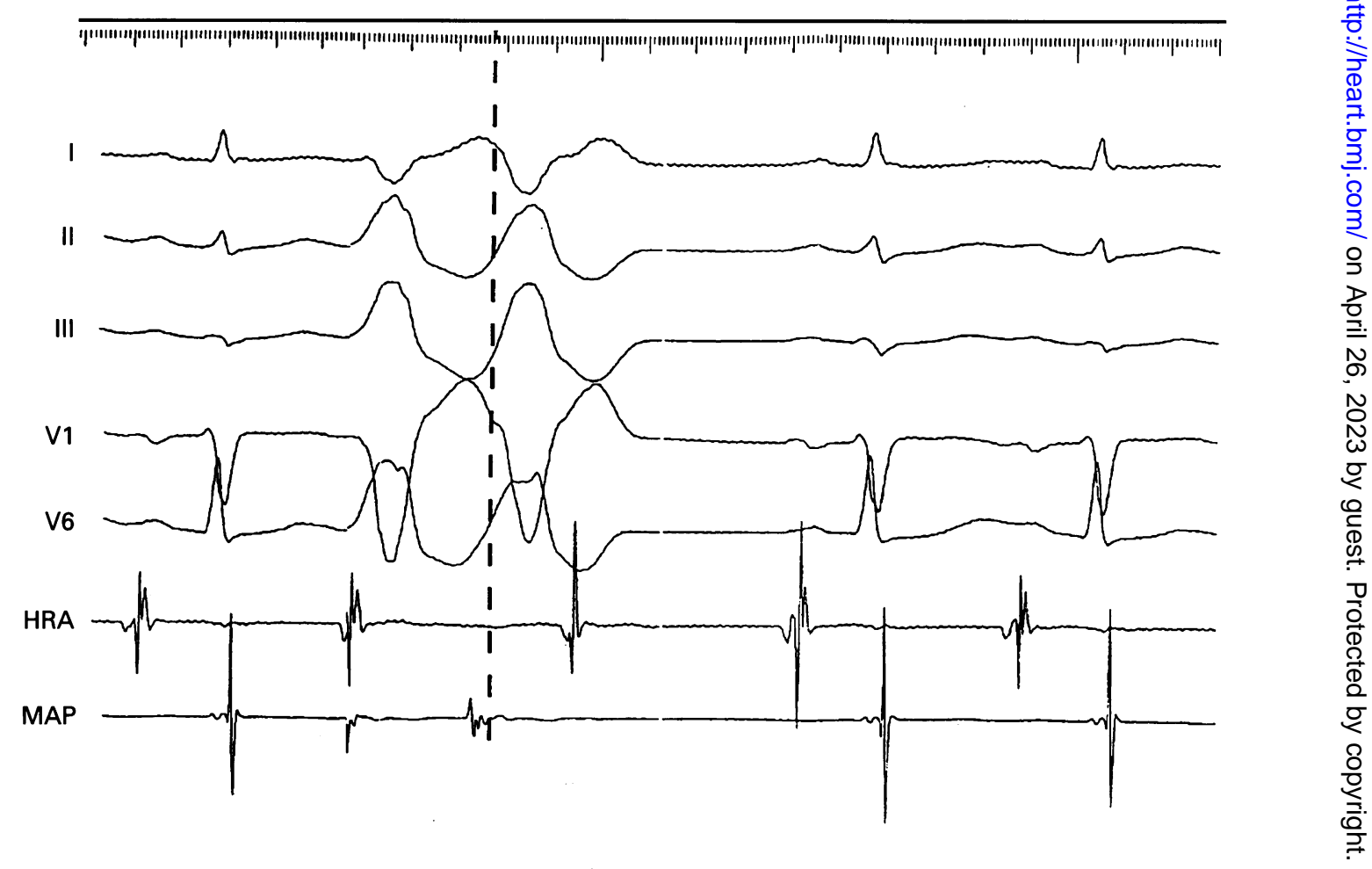

Figure 2 Local electrograms recorded from the distal poles of the ablation catheter at the successful target site. Only two ventricular beats are seen the second more closely resembles the spontaneous tachycardia morphology, in particular the $Q R S$ configuration in lead V6. Because catheter pressure at the ablation site suppressed longer runs of spontaneous non-sustained ventricular tachycardia, there are no local electrogram recordings obtained during a long run of tachycardia. The dashed line shows the onset of the QRS complex, indicated by the notch on the T wave in lead VI. The local electrogram began about $30 \mathrm{~ms}$ before the onset of the $Q R S$ complex. The large time lines indicate 1 second intervals. 
mic medication, and exercise tolerance returned to normal. The ejection fraction determined by radionuclide angiography at one year after the ablation was $61 \%$ and left ventricular chamber size and wall motion were normal.

\section{Discussion}

To our knowledge this is the first report published in English of the successful transcatheter cure of ventricular tachycardiainduced cardiomyopathy. This patient had incessant right ventricular outflow tract tachycardia and substantial left ventricular dysfunction. The ventricular tachycardia and left ventricular dysfunction resolved with successful radiofrequency ablation. The long duration of symptoms of palpitations and the rapid recovery of left ventricular function argue against a transient phenomenon such as myocarditis being responsible for both the left ventricular dysfunction and the tachycardia. One earlier report described the successful catheter ablation of ventricular tachycardia in a patient with tachycardia-induced cardiomyopathy and severe left ventricular dysfunction by direct current (DC) energy, resulting in normalisation of left ventricular function and size. ${ }^{5}$ The duration and rate of tachycardia required to produce tachycardia-induced cardiomyopathy may be vary. In most earlier reports, ${ }^{15}$ the tachycardia producing cardiomyopathy has been persistent. In this patient, tachycardia was catecholamine sensitive, was absent at night, and present only intermittently during the day. This suggests that tachycardia that does not affect all cardiac cycles can induce myopathy.

When cardiomyopathy and ventricular tachycardia are identified simultaneously it may not be possible to define a cause and effect relation. In this case the rapid resolution of the left ventricular dysfunction that followed successful ablation of ventricular tachycardia favours the tachycardia as a cause of cardiomyopathy. Although this diagnosis was suspected before catheter ablation, it was not possible to confirm the presence of tachycardia-induced cardiomyopathy until after the left ventricular dysfunction was shown to be reversible.

In summary, incessant ventricular tachycardia can cause reversible left ventricular dysfunction. The diagnosis should be suspected in patients who present with incessant tachycardia and apparent idiopathic cardiomyopathy. Successful radiofrequency ablation can resolve the tachycardia and the left ventricular dysfunction.

1 Packer D, Bardy G, Worley S. Tachycardia-induced cardiomyopathy: a reversible form of left ventricular dys function. Am 7 Cardiol 1986;57:563-70.

2 Cruz F, Cheriex E, Smeets J. Reversibility of tachycardiainduced cardiomyopathy after cure of incessant supra ventricular tachycardia. $\mathcal{F} \mathrm{Am}$ Coll Cardiol 1987;16: 739-44.

3 Williams R, Kass D, Kawagoe Y, Pak P, Tunin RS, Sha $R$, et al. Endocardial gene expression during developmen of pacing tachycardia induced heart failure in the dog. Circ Res 1994;75:615-23.

4 Fyfe D, Gillette P, Crawford F. Resolution of dilated cardiomyopathy after surgical ablation of ventricular tachycardia in a child. $\mathcal{F}$ Am Coll Cardiol 1987;9:231-4.

5 Matsuura Y, Chin W, Kurihara T. Tachycardia-induced cardiomyopathy. A case report. $\mathcal{F}$ Cardiol 1990;20: 509-18.

6 Wen MS, Yeh SJ, Wang CC, Lin FC, Chen IC, V Wu Radiofrequency ablation therapy in idiopathic left venRadiofrequency ablation therapy in idiopathic left ven-
tricular tachycardia with no obvious structural heart districular tachycardia with no obvio 